\title{
The effect of pravastatin on obstetrics complications associated with uteroplacental insufficiency: A systematic review and meta-analysis
}

\author{
Ayala Hirsch ${ }^{1}$, Natali Ternovsky ${ }^{2}$, Reut Rotem ${ }^{1}$, and Bruria Hirsh Raccah ${ }^{2}$ \\ ${ }^{1}$ Shaare Zedek Medical Center \\ ${ }^{2}$ Hebrew University of Jerusalem
}

January 25, 2022

\begin{abstract}
ABSTRACT Background: Obstetrical complications involving uteroplacental insufficiency associated disorders, such as preeclampsia, intrauterine growth restriction, and obstetric antiphospholipid syndrome, share pathophysiology and risk factors with cardiovascular diseases treated with statins. Objective: To evaluate associations of statin treatment with pregnancy prolongation, and neonatal and maternal morbidity, among women with uteroplacental insufficiency disorders. Search Strategy: Electronic databases including PubMed, Medline, Embase, Clinical Trials Registry Clinicaltrials.gov, and The Cochrane Library were searched from inception to January 2022. Selection Criteria: Cohort studies and randomized controlled trials (RCTs) Data collection and analysis: Pooled odds ratios were calculated using a random-effects model; meta-regression was utilized when applicable. Main Results: The analysis included ten studies describing 1391 women with uteroplacental insufficiency-associated disorders: 703 treated with pravastatin and 688 not treated with statins. Women who received pravastatin showed significant prolongation of pregnancy (mean difference 0.44 weeks, 95\% CI:0.01-0.87, $\mathrm{p}=0.04, \mathrm{I} 2=96 \%$ ) and less neonatal critical care unit admission $(\mathrm{OR}=0.42,95 \% \mathrm{CI}: 0.23-0.75, \mathrm{p}=0.004, \mathrm{I} 2=25 \%)$. Trends were observed toward a decrease in preeclampsia diagnoses $(\mathrm{OR}=0.51,95 \% \mathrm{CI}: 0.25-105, \mathrm{p}=0.07, \mathrm{I}=44 \%)$, and perinatal death $(\mathrm{OR}=0.32,95 \% \mathrm{CI}: 0.09-1.13, \mathrm{p}=0.08, \mathrm{I} 2=54 \%)$ and an increase in birth weight (mean difference $=102$ grams, 95\%CI: $-14-212, \mathrm{p}=0.08, \mathrm{I} 2=96 \%$ ). A meta-regression analysis revealed associations between earlier gestational age at initiation of pravastatin treatment to lower risk for development of preeclampsia $(\mathrm{R} 2=1)$ and between longer duration of pravastatin treatment to lower rate of NICU admission (R2=0.33). No dose-response effect was demonstrated. Conclusions: Pravastatin treatment in pregnancies with high risk for developing uteroplacental insufficiency disorders may prolong pregnancy duration and improve neonatal outcomes.
\end{abstract}

\section{Introduction}

Preeclampsia and intrauterine growth restriction (IUGR) are serious complications of pregnancy that increase the morbidity and mortality of foetuses and parturients. Both these complications have been associated with the development of placental insufficiency. Abnormal placentation and its associated disorders may be encountered among parturients with obstetric antiphospholipid syndrome; 20\%-30\% of pregnancies among these parturients may result in preeclampsia, IUGR and foetal losses. The spectrum of uteroplacental insufficiency disorders results from various factors, including poor trophoblast uterine invasion early in pregnancy, impaired transformation of the uterine spiral arteries to high capacity-low impedance vessels, abnormalities in the development of chorionic villi, endothelial dysfunction and pathologic changes in the antiangiogenic environment. Prevention strategies for uteroplacental insufficiency are not currently known. Therefore, preeclampsia or IUGR occurring early in the course of pregnancy prompts early delivery, which can lead to neonatal death and disability arising from prematurity, and also maternal morbidity.

The role of statins in treating and preventing cardiovascular diseases is well established; their potential benefit in treating multiple non-cardiovascular outcomes is currently under investigation. Endothelial dysfunction in preeclampsia, IUGR and obstetric antiphospholipid syndrome has been associated with abnormalities in 
lipid profile, high levels of triglycerides and oxidative stress. statins inhibit HMG-CoA reductase, leading to reduced plasma cholesterol levels. statins also have antioxidant, anti-inflammatory, anti-thrombogenic and vasodilating effects. The preclinical evidence supporting the use of statins in the treatment of preeclampsia has been demonstrated in multiple animal models.

Current clinical knowledge regarding the efficacy of statins in the treatment of preeclampsia is inadequate. Individual studies have presented contradicting results. Accordingly, current guidelines do not recommend the usage of statins in the prevention or treatment of uteroplacental insufficiency disorders.

The aim of this systematic review and meta-analysis was to assess pregnancy outcomes associated with pravastatin treatment, among pregnancies with high risk for development of uteroplacental insufficiency and pregnancies that already developed associated disorders: preeclampsia and IUGR, with or without obstetric antiphospholipid syndrome.

\section{Methods}

\section{Search strategy}

This systematic review followed the Preferred Reporting Items for Systematic Reviews and Meta-Analyses (2020) framework guidelines (PRISMA) and the Meta-analysis Of Observational Studies in Epidemiology (MOOSE) guidelines. (Supplement table S1-S2)

We conducted three systematic database searches: the first included articles from 1953 to February 2020, the second included articles published from the last search to April 2021 and the third included articles published from the last search to January 2022. The search included PubMed/Medline, Embase, Clinical Trials Registry Clinicaltrials.gov and The Cochrane Library. Language restrictions were not set.

The search strategies incorporated index terms (Mesh) and free text words for the search concepts: pravastatin, atorvastatin, rosuvastatin, pregnancy combined by "AND"; and in each domain, the terms were combined by "OR" (Supplementary table S3) The first domain contained terms on statins (including synonyms and abbreviations such as HMG-CoA reductase inhibitors), the second domain related to pregnancy.

The detailed protocol is documented online in the International Prospective Register of Systematic Reviews Registry (CRD42020165804). Because this study was a review and meta-analysis, Helsinki board approval was waived.

Data sources and searches

In the search strategy, we included randomized controlled trials (RCTs), non-randomized controlled clinical trials, prospective and retrospective comparative cohort studies, and case-control studies. Every study that included women treated with statins during pregnancy was analysed. Duplicated reports, case reports, case series, cross-sectional studies, pharmacokinetic studies in healthy adults, animal studies, reviews, expert opinion, editorials, letters to the editor, comments, and studies with a high risk of bias were excluded.

Study selection and data extraction

Two investigators (A.H. and N.T.) independently identified and extracted articles for potential inclusion, using the Rayyan QCRI web application for systematic reviews. Disagreements were resolved by referral to a third reviewer (B.H.R.). The full texts of the resulting references were retrieved and analysed. If more than one study published data from the same cohort, we included the report with the higher quality according to the Risk of bias In Non-randomized Studies of Interventions (ROBINS-I) tool (Newcastle-Ottawa Quality Assessment Scale- NOS) to avoid overlap.

Exposure to statins during pregnancy was defined as exposure to any dose and in any trimester of pregnancy. Pregnancies that were defined as high risk for future development of uteroplacental insufficiency disorder and those that had developed uteroplacental insufficiency disorders were included. The spectrum of uteroplacental insufficiency disorders included: preeclampsia, early-onset IUGR $(<28 \mathrm{w})$, and obstetrical antiphospholipid syndrome. Multiple pregnancies were excluded in patient-level data to avoid bias. The 
primary outcome included prolongation of pregnancy from study entry to delivery. The secondary outcomes included neonatal outcomes such as admission to the neonatal intensive care unit (NICU), birth weight and neonatal death, and the maternal occurrence of a new diagnosis of preeclampsia. Neonatal death was defined as stillbirth or death in the first month of life. Data were extracted from the included studies by a single reviewer and subsequently evaluated by the second reviewer. For studies that did not report the outcomes, we contacted the authors and requested the missing data.

Quality assessment and the risk of bias

The risk of bias and the quality of the observational studies were assessed using the Newcastle-Ottawa Quality Assessment Scale (NOS). The scale is based on eight criteria and provides a score ranging from 0 (high risk of bias) to 9 stars (low risk of bias). A 5-star rating and below was designated high risk of bias, 6-7 stars intermediate risk of bias, and 8-9 stars low risk of bias. Randomized controlled studies were evaluated by the Cochrane Collaboration's Risk of Bias Tool. Summary assessments of the risk of bias were derived for each study. Assessments were carried out independently by two investigators (A.H and N.T.).

Data synthesis and analysis

Meta-analysis and meta-regression were performed using Comprehensive Meta-Analysis software. Randomeffect pooled odds ratios (ORs) were calculated with the corresponding $95 \%$ confidence intervals (CIs) to summarize the results overall and within subgroups. Heterogeneity was assessed by using the $\mathrm{I}^{2}$ statistic. We performed subgroup analyses by study type (RCT, cohort). Random-effects meta-analysis was used to pool analyses. Meta-regression analyses were performed to evaluate whether differences in pravastatin dosage and timing of treatment initiation modified the association between exposure to pravastatin and outcomes, and to explain the heterogeneity in the estimated effect size. The data were analyzed using the Comprehensive Meta-Analysis software.

\section{Publication bias}

Assessment was planned, of publication bias by visual inspection of the funnel plot, provided the analysis would include at least ten studies.

\section{Results}

\section{Study selection}

The database search yielded 3336 citations: PubMed $(\mathrm{n}=636)$, Embase $(\mathrm{n}=1989)$, Cochrane Library $(\mathrm{n}=$ $621)$, clinicaltrials.gov $(\mathrm{n}=90)$. In total, 667 were identical duplicates, and excluded. After abstract assessment, 62 articles were extracted for full-text review. Ten articles dropped out because they included normal pregnancies without any risk factors, nor signs or symptoms that indicated placental insufficiency.. One study was excluded because of wrong outcomes and another was excluded due to lack of control group . Fourteen articles were excluded because of publication type or study design. Studies involving animals or human placenta were excluded, only clinical studies were included. Due to a lack of standardization in studies investigating biomolecular markers, those studies or results were excluded as well. The selection process is illustrated in Figure 1. Ultimately, ten studies were included in the analysis, with a total of 1391 pregnant women. Of them, $703(50.5 \%)$ were treated with pravastatin and $688(49.5 \%)$ were not treated with statins. All ten studies described high treatment adherence.

\section{Characteristics of the studies}

The studies were published between 2016 and 2021 and originated from the United States $(\mathrm{n}=2)$, Indonesia $(n=2)$, Serbia $(n=2)$, UK $(n=1)$, Spain $(n=1)$, Greece $(n=1)$, and multicentre over Europe $(n=1)$. Four were cohort studies and six RCTs. Studies included women with uteroplacental insufficiency defined as early onset of preeclampsia between gestational age 24 and 31 weeks or high risk for developing preeclampsia. This risk was defined in two studies as a history of severe preeclampsia in a prior pregnancy that required delivery before 34 weeks gestation. In another study, as a history of previous preeclampsia and birth before 37 weeks of gestation or at least two main risk factors for preeclampsia. One study selected women with at 
least $20 \%$ risk for developing preeclampsia based on the presence of minimally 2 independent clinical risk factors or abnormal doppler velocimetry index. and another selected women with high risk for preeclampsia based on a survival-time model of Bayes theorem . Two studies defined uteroplacental insufficiency as a pregnancy with an obstetric antiphospholipid syndrome that developed preeclampsia or IUGR associated with abnormal placental Dopplers. An additional study defined a pregnancy with uteroplacental deficiency as having a past poor obstetric history and abnormal placental Dopplers. Abnormal placental Dopplers was defined as uterine artery pulsatility index above the 95 th percentile. One study also included the early onset of IUGR, diagnosed earlier than 28 weeks of pregnancy.

Although the search algorithm included all types of statins, only pravastatin was used in the studies included. The dosage of pravastatin differed between studies: $40 \mathrm{mg}$ in five studies, $20 \mathrm{mg}$ in four studies and $10 \mathrm{mg}$ in one study. The comparator treatment included aspirin together with low molecular weight heparin in two studies, aspirin alone in one study, placebo in four studies and no treatment in two studies. Most studies initiated treatment with pravastatin in the second trimester, but in two studies a minority of the patients were diagnosed and started treatment in their third trimester and one large RCT included only women in their late third trimester. Table 1 shows a summary of the key characteristics of the included trials.

Quality assessment

The overall risk of bias among the four nonrandomized studies according to the Newcastle-Ottawa Quality Assessment Scale (NOS) was 7.5. The overall risk of bias of the six randomized controlled studies evaluated by the Cochrane Collaboration's Risk of Bias Tool was low. The risk of bias assessment is summarized in Figures S1 and S2.

As ten studies were included in the meta-analysis, we were able to test for funnel plot asymmetry to assess possible publication bias. Visual inspection of the funnel plots revealed no indication of publication bias, Egger test also indicates no statistical significance in asymmetry $(\mathrm{p}=0.5)$. (Figure S3).

Synthesis of results

Pregnancy prolongation :

Eight studies compared the difference in prolongation of pregnancy from study entry to delivery between women treated with pravastatin and women not treated. Pravastatin treatment was associated with a significant prolongation of pregnancy from study entry to delivery of 0.44 weeks (mean difference 0.44 weeks, 95\%CI:0.01-0.87, $\mathrm{p}=0.04, \mathrm{I}^{2}=96 \%$ ) (Figure 2).

\section{NICU admission:}

Nine studies examined NICU admission with or without pravastatin treatment. Pravastatin treatment was associated with a significantly decreased risk for NICU admission compared to women not treated with statins ( $\mathrm{OR}=0.42,95 \% \mathrm{CI}$ : $0.23-0.75, \mathrm{p}=0.004, \mathrm{I}^{2}=25 \%$ ) (Figure 3 ).

Perinatal death:

Ten studies examined the association of pravastatin with perinatal death. Although statistical significance was not reached, there was a trend toward a decrease in the risk for perinatal death in newborns of women treated with pravastatin $\left(\mathrm{OR}=0.32,95 \% \mathrm{CI}: 0.09-1.13, \mathrm{p}=0.08, \mathrm{I}^{2}=54 \%\right.$ ) (Figure 4$)$.

\section{Birth weight:}

Eight studies compared birth weight between newborns of women treated with pravastatin and those who did not. The increase in birth weight following pravastatin treatment was not statistically significant (Mean difference $=102$ grams, 95\%CI: $-14-212, \mathrm{p}=0.08, \mathrm{I}^{2}=96 \%$ ) (Figure 5).

\section{Maternal outcomes:}

Six studies described a new diagnosis of preeclampsia after initiation of pravastatin treatment. Among women treated with pravastatin compared to control groups, the risk for the development of preeclampsia 
was non significantly decreased $(\mathrm{OR}=0.51,95 \% \mathrm{CI}: 0.25-105, \mathrm{p}=0.07, \mathrm{I}=44 \%$ ) (Figure 6).

In a sensitivity analysis that excluded Döbert et al study who selected women only in their late third trimester, The risk for the development of preeclampsia was significantly decreased (OR=0.37, 95\%CI:0.18$0.74, \mathrm{p}=0.01, \mathrm{I}=0 \%$ ) (Figure 7 ).

\section{Meta-regression:}

Due to the high heterogeneity found in the analyses, a meta-regression analysis was conducted. A metaregression revealed a statistically significant association between early gestational age at initiation of pravastatin treatment and decreased risk for the development of preeclampsia $\left(\mathrm{R}^{2}=1\right)$ and NICU admission $\left(\mathrm{R}^{2}=0.33\right)$. Statistically significant associations were not found between higher pravastatin dose to prolongation of pregnancy from study entry to delivery, birth weight, perinatal death or NICU admission. Statistically significant associations were not found between earlier gestational age at initiation of pravastatin treatment to prolongation of pregnancy from study entry to delivery, birth weight or perinatal death (Figure S4-S13).

\section{Discussion}

\section{Main findings}

In this meta-analysis and meta-regression, pravastatin usage for uteroplacental insufficiency was associated with a significant prolongation of pregnancy, mean 0.44 weeks, from study entry to delivery. Prematurity is the main cause of neonatal mortality, and early neonatal as well as late childhood morbidity. Preventing preterm births has been delineated as one of the most urgent goals in current obstetrics. Prolonging pregnancy has most probably resulted in the significant decrease observed in NICU admission, and the trends towards decreased perinatal death and increased neonatal birth weight. In addition, a sensitivity analysis that excluded Döbert et al study, that selected women only in their late third trimester, new onset of preeclampsia was less common under pravastatin treatment.

Comparison with existing literature

The similarities in pathophysiology and risk factors shared by uteroplacental insufficiency disorders and cardiovascular disease have prompted the search for treatments of uteroplacental insufficiency with agents used to treat vascular disease. The underlying mechanism of both conditions involves vascular endothelial dysfunction with endothelial inflammation. The American Heart Association included a history of preeclampsia as a risk factor for future cardiovascular disease. This association might relate to the shared risk factors for cardiovascular diseases preceding pregnancy, such as obesity, hypertension, diabetes, and dyslipidaemia or alternatively, to the metabolic and vascular changes that preeclampsia itself induces.

Numerous clinical studies have confirmed the multiple therapeutic benefits resulting from the pleiotropic effects of statins. Statins improve endothelial dysfunction by protecting vascular endothelium and stimulating its regeneration and its angiogenesis. The antioxidant action of statins relates to their antithrombotic and vasodilating activities and inhibition of free radical formation. The anti-inflammatory action of statins results from their impact on the immune response, which is expressed as increased blood level inflammatory markers and mediators. The latter include C-reactive protein, L-, E- and P-selectin, intercellular and vascular adhesion molecule and Hmox-1, which inhibits sFlt-1 and sEng. According to this pathophysiology, statins have been shown to directly improve endothelial dysfunction and angiogenic imbalance in human placenta. Thus, statins may be a promising agent in preventing and treating uteroplacental dysfunction.

The safety profile of statin exposure during pregnancy is not well defined. United States Food and Drug Administration labelling recommends against the use of statins during pregnancy, based on animal data showing teratogenic potential at high doses. Therefore, the current practice encompasses the advice to discontinue statins when trying to conceive. However, subsequent case registries did not demonstrate an association between congenital anomalies and statin exposure. The clinical benefits of statins in individuals with familial hypercholesterolemia or cardiovascular diseases should be considered, together with the growing evidence of statins' potential benefit in preventing and treating uteroplacental insufficiency-associated 
disorders. Accordingly, their benefit may fairly overcome their controversial risk.

Strengths and limitations

To the best of our knowledge, this is the largest and the only study to pool results of pravastatin for uteroplacental insufficiency-associated disorders. This meta-analysis included all available published data and conducted a systematic analysis according to accepted guidelines. Six randomized control trials were included, and all ten studies described high treatment adherence.

As four studies included in this meta-analysis were retrospective, selection bias should be considered. We performed a quality assessment for the risk of bias using the NOS, which resulted in a low probability of selection bias. Due to the small numbers of studies and events, our results should be interpreted with caution, and further studies are required. The cohorts included in this meta-analysis were geographically diverse. This can potentially broaden the generalizability of our results yet highlights difference in management and background characteristics.

The potential weakness of this analysis rests in the high heterogeneity among the studies, and the inclusion of only six RCTs, with a small number of patients. The favourable outcomes of this meta-analysis are largely driven by "cohort" studies which may have included biases, as the women included in the control groups represented very specific populations. Yet, to address this heterogeneity, a sub-group analysis and random effect model was used. High heterogeneity was also viewed in dosage regimes, treatment indications and the lack of standardization. To address the high heterogeneity, we conducted a meta-regression for pravastatin dosage, study type and early vs late initiation of pravastatin treatment. Although the analyses of perinatal death and birth weight showed trends toward better outcome, these trends were not statistically significant. This might also be attributed to the high heterogeneity and the small events in the studies.

The mutual mechanism of uteroplacental dysfunction justifies the shared analysis. However, while the pathophysiology of uteroplacental dysfunction lies in inadequate trophoblast invasion in the early stages of pregnancy, all the studies that investigated pravastatin therapy started in the second to the third trimester and the largest study included only parturients in late third trimester which might downgrade the accepted results. Earlier initiation of therapy might yield different results from those described. Biomolecular markers were measured in few studies. However, biases may have arisen due to lack of standardization of measurements performed over various periods of time, and the exclusion from the analysis of women who delivered after the first measurement. In addition, no data were found in the literature on other potent statins such as atorvastatin and rosuvastatin: this could also have affected the results.

In this analysis, we pooled studies that adjusted for confounders including pre-existing diabetes, a baseline score of biomolecule markers and laboratory findings; together with studies that did not perform any adjustments.' This raises the possibility that our results may not reflect the true effect size and may be susceptible to sources of bias. Although the studies are heterogeneous, as they represent real-life conditions, we decided to pool them together.

Conclusion

This meta-analysis suggests that treatment with pravastatin may prolong pregnancy duration in women with uteroplacental insufficiency disorder. An individualized assessment and a cautious approach are advised if pravastatin usage is considered.

\section{Conflicts of interest-none}

\section{Acknowledgment- none}

\section{Contribution to authorship}

All authors contributed to the study protocol. The literature search was performed by A.H, N.T and B.H.R. Literature screening, data extraction, were performed by D.P, A.H, N.T and B.H.R. Risk of bias evaluation was performed by A.H and N.T. 
A.H and B.H.R conducted the analyses. The draft of the manuscript was written by A.H, B.H.R and R.R., and all authors commented on earlier versions of the manuscript. All authors read and approved the final manuscript

Disclosure statement: The authors report no conflict of interest.Source of funding: None

Acknowledgments: Not applicable

\section{References}

Table 1 :Methodologic characteristics of the included studies

\section{Figures:}

Figure 1: Publication selection process .

Figure 2: Meta-analysis results of the association of pravastatin treatment with a mean difference (weeks) in prolongation of pregnancy from study entry to delivery.

Figure 3: Meta-analysis results of the association of pravastatin treatment with admission to the neonatal intensive care unit.

Figure 4: Meta-analysis results of Odds ratios for perinatal death following pravastatin treatment versus a control group.

Figure 5: Meta-analysis results of the Differences in birth weight (grams) following pravastatin treatment versus a control group.

Figure 6: Meta-analysis results of the association of pravastatin treatment with new diagnoses of preeclampsia.

Figure 7: Sensitivity meta-analysis of the association of pravastatin treatment with new diagnoses of preeclampsia.

\section{Hosted file}

TABLE1_jan 15. docx available at https://authorea.com/users/338183/articles/554012-the-effectof-pravastatin-on-obstetrics-complications-associated-with-uteroplacental-insufficiencya-systematic-review-and-meta-analysis 


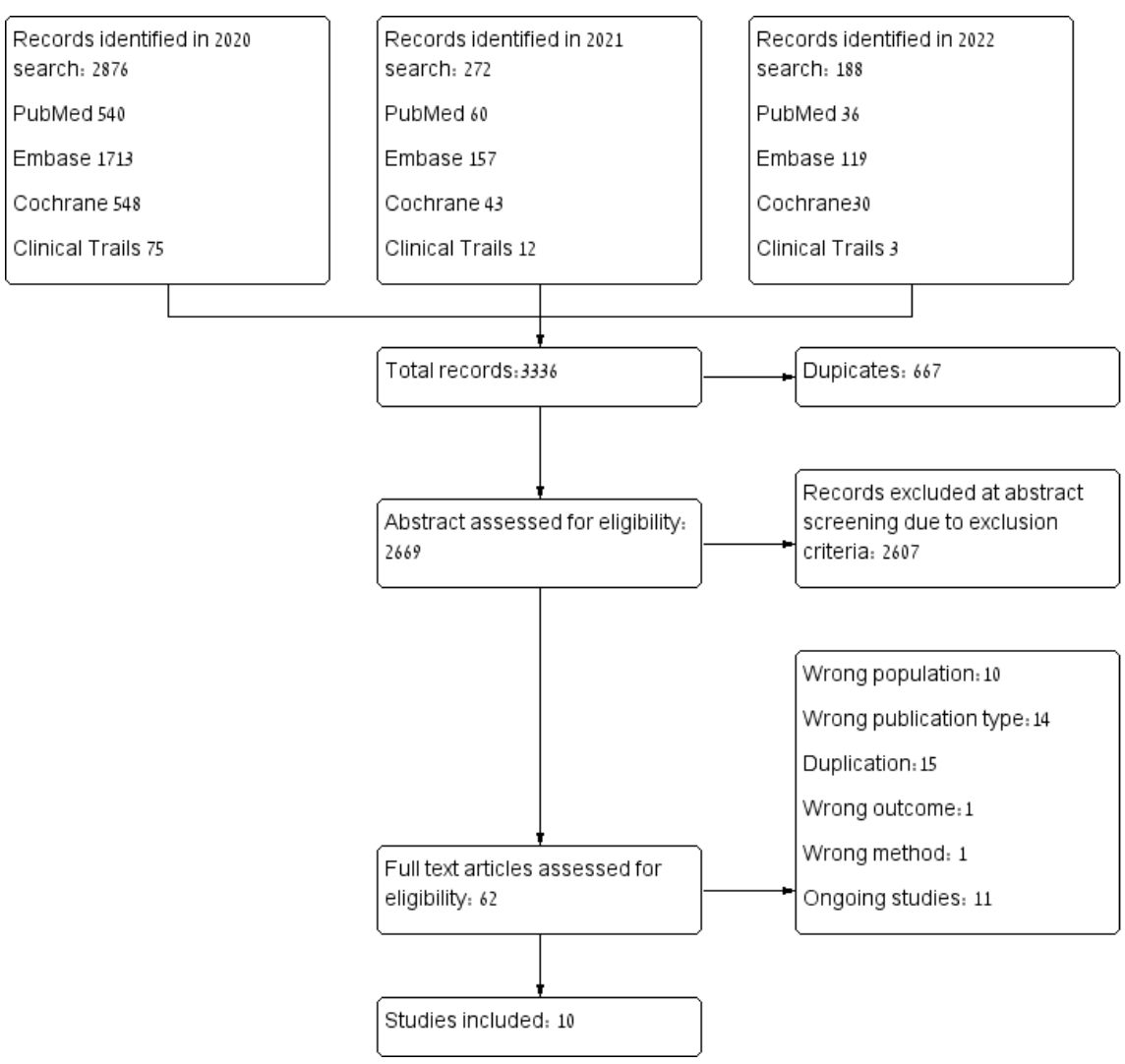

Figure 2: Meta-analysis results of the association of pravastatin treatment with a mean difference (weeks) in prolongation of pregnancy from study entry to delivery.

Study name Statistics for each study Difference Lower Upper
in means limit limit

Cohort Lefkou E, 2016

Mendoza M, 2020

Jurisic A, 2021

Lefkoua E, 2020

RCT AhmedA, 2019

Costantine MM, 201

Costantine MM, 202

Akbar, 2021

$7.80 \quad 5.85$

$\begin{array}{rrrr}7.20 & -1.80 & 6.20 & 0.282\end{array}$

$\begin{array}{llll}14.50 & 11.61 & 17.39 & 0.000\end{array}$

$\begin{array}{llll}11.00 & 6.51 & 15.49 & 0.000\end{array}$

$\begin{array}{llll}8.93 & 4.22 & 13.65 & 0.000\end{array}$

$\begin{array}{llll}0.30 & -0.15 & 0.75 & 0.19\end{array}$

$\begin{array}{llll}1.50 & -0.84 & 3.84 & 0.210\end{array}$

$\begin{array}{llll}1.20 & -1.04 & 3.44 & 0.293\end{array}$

$\begin{array}{llll}0.00 & -5.22 & 5.22 & 1.000\end{array}$

$\begin{array}{lllll}0.37 & -0.06 & 0.80 & 0.09\end{array}$

$\begin{array}{llll}0.44 & 0.01 & 0.87 & 0.04\end{array}$



Favours Pravatatin Favours Control

CI, confidence interva 
Figure 3: Meta-analysis results of the association of pravastatin treatment with admission to the neonatal intensive care unit

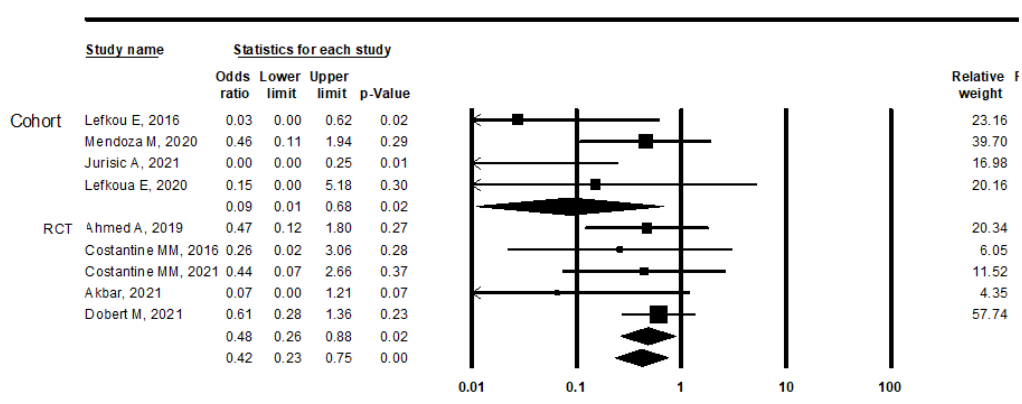

Favours Pravastatin Favours Control

CI, confidence interval

Figure 4: Meta-analysis results of Odds ratios for perinatal death following pravastatin treatment versus a control group

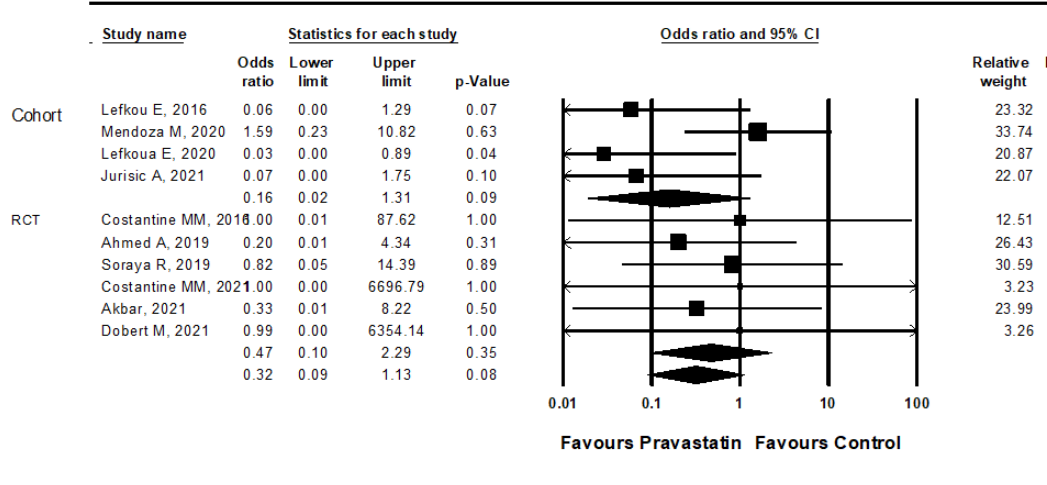

CI, confidence interval

Figure 5 : Meta-analysis results of the Differences in birth weight (grams) following pravastatin treatment versus a control group

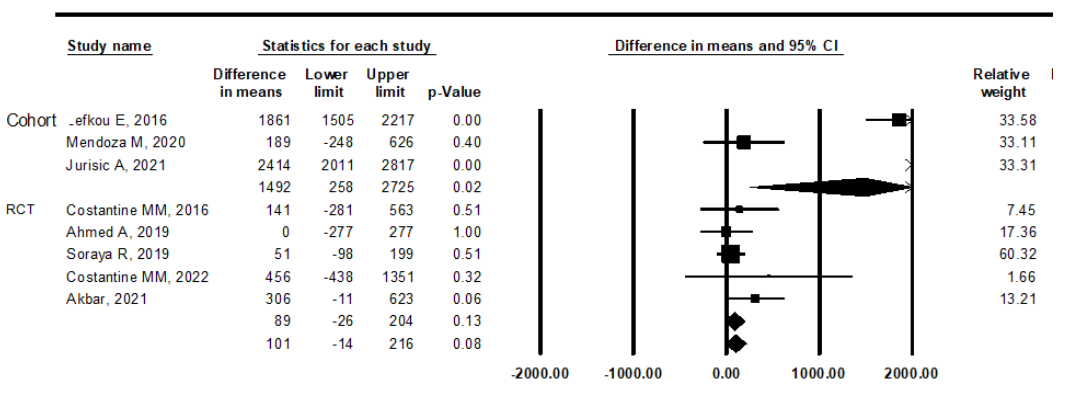

Favours Pravastatin Favours Control

CI, confidence interval 
Figure 6: Meta-analysis results of the association of pravastatin treatment with new diagnoses of preeclampsia

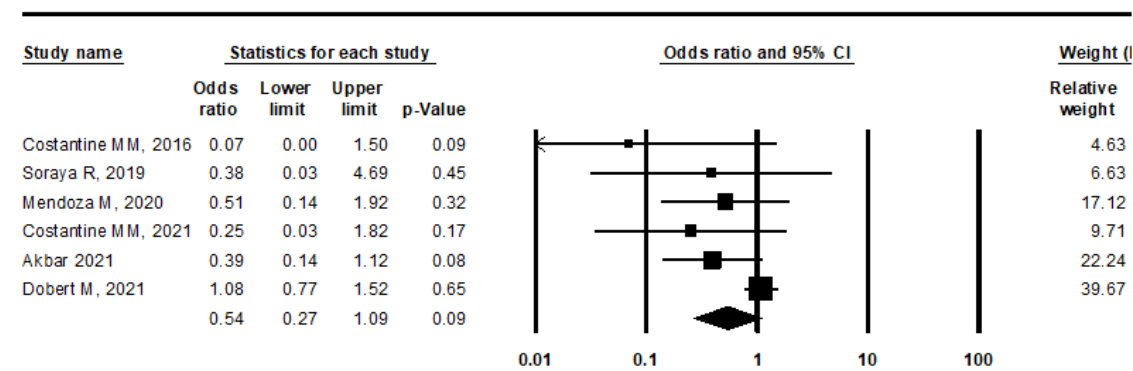

Favours Pravastatin Favours Control

CI, confidence interval

Figure 7: Sensetivity Meta-analysis results of the association of pravastatin treatment with new diagnoses of preeclampsia

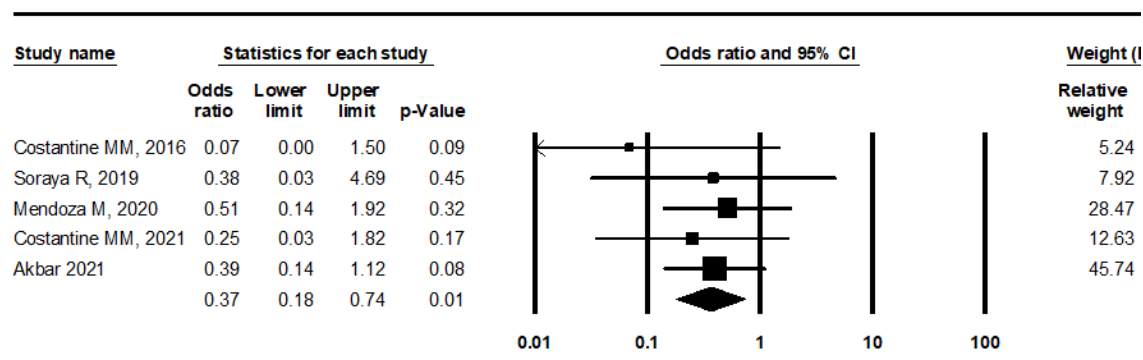

Favours Pravastatin Favours Control

CI, confidence interval 